Research Paper

\title{
Liquid biopsy in colon cancer: comparison of different circulating DNA extraction systems following absolute quantification of KRAS mutations using Intplex allele-specific PCR
}

\author{
Vera Kloten ${ }^{1}$, Nadine Rüchel ${ }^{1}$, Nadina Ortiz Brüchle ${ }^{1}$, Janina Gasthaus ${ }^{1}$, Nils \\ Freudenmacher ${ }^{1,2}$, Florian Steib ${ }^{1}$, Jolein Mijnes $^{1}$, Julian Eschenbruch ${ }^{1}$, Marcel \\ Binnebösel $^{3}$, Ruth Knüchel ${ }^{1}$ and Edgar Dahl ${ }^{1,2}$ \\ ${ }^{1}$ Molecular Oncology Group, Institute of Pathology, University Hospital Aachen, Aachen, Germany \\ ${ }^{2}$ Centralized Biomaterial Bank of RWTH Aachen University (RWTH cBMB), Institute of Pathology, University Hospital Aachen, \\ Aachen, Germany \\ ${ }^{3}$ Department of Visceral and Transplantation Surgery, University Hospital Aachen, Aachen, Germany \\ Correspondence to: Vera Kloten, email: vkloten@ukaachen.de \\ Keywords: liquid biopsy, KRAS, cfDNA extraction systems, ctDNA quantification, intplex-allele specific PCR \\ Received: November 30, $2016 \quad$ Accepted: August 05, $2017 \quad$ Published: September 21, 2017 \\ Copyright: Kloten et al. This is an open-access article distributed under the terms of the Creative Commons Attribution License 3.0 \\ (CC BY 3.0), which permits unrestricted use, distribution, and reproduction in any medium, provided the original author and source \\ are credited.
}

\section{ABSTRACT}

Non-invasive molecular analysis of circulating tumor DNA (ctDNA) is a promising application in personalized cancer management, although there is still much to learn about the biological characteristics of ctDNA. The present study compared absolute amounts of KRAS mutated ctDNA and total circulating cell-free DNA (cfDNA) in colorectal cancer $(C R C)$ patients $(n=50)$ from various stages and healthy controls $(n=8)$ by Intplex allele-specific and digital droplet PCR. In addition, the impact of two prominent extraction techniques (silica-based membrane vs. magnetic beads) on cfDNA and ctDNA recovery was analyzed in $\mathbf{3 8}$ paired samples from CRC patients and specific spike-in DNA controls. CfDNA fragment size was assessed using the Agilent 2100 Bioanalyzer. Relative quantities of total cfDNA quantities were measured using the Qubit fluorometer. Statistical analysis on total cfDNA yield revealed a strong correlation $(r=0.976)$ between Qubit and absolute Intplex allele-specific PCR measurements in cancer patients and healthy controls. Total cfDNA was significantly increased in cancer patients compared to healthy controls, with the highest yield in distant metastatic disease. In line, the highest amount of ctDNA $(1.35 \mathrm{ng} / \mu \mathrm{L})$ was found in patients with distant organ metastasis. Of great interest, the silica-based membrane method significantly promoted extraction of long cfDNA fragments. In contrast, the magnetic bead system more efficiently recovered short cfDNA fragments in serum of cancer patients. Further, a decreased KRAS allele frequency was observed in serum compared to plasma. This study suggests that the source of cfDNA and choice of pre-analytical extraction systems needs to be more carefully validated in routine clinical practice.

\section{INTRODUCTION}

Blood-based molecular analysis of tumor-derived circulating DNA (ctDNA) is becoming an established tool for monitoring tumor burden and detection of resistance early during targeted cancer therapies [1-3]. In contrast to total free circulating DNA (cfDNA) levels, i.e. circulating DNA from different cells-of-origin like healthy, malignant and tumor microenvironmental cells, quantity of ctDNA harboring cancer-associated aberrations is stage- and entity-dependent [4]. In addition, Bettegowda and colleagues already revealed an extreme variability of mutated ctDNA fragments in e.g. colon cancer patients (between 1 and 100.000 mutated fragments) illustrating 
the need for highly sensitive detection techniques. Innovative technologies like digital and allele-specific PCR were shown to detect oncologic point mutations (e.g. $E G F R$ or $K R A S$ ) with a sensitivity of $0.01 \%$, i.e. 2 mutant allele copies in a background of 20.000 wild type alleles $[5,6]$. However, recent studies investigating the secondary EGFR T790M resistance mutation in advanced non-small cell lung cancer (NSCLC) patients showed that about 20$30 \%$ of tissue T790M mutations were not detectable in plasma-ctDNA, even with highly sensitive digital PCR technologies [7]. While ctDNA-based detection of the $E G F R$ T790M mutation is already an approved diagnostic application in those cases where no tissue biopsy is available, it becomes clear that recent developments in liquid biopsy are rather based on innovative technologies than on an established knowledge about the analytes themselves.

The variability of mutated ctDNA fragments in cancer patients provides evidence that the biology of different cancer (sub-) types may be a limiting factor for detecting ctDNA mutations. Thus, a better knowledge on size and fragmentation are necessary for reliable quantification and analysis on circulating DNA. Various studies on circulating DNA illustrated a characteristic apoptotic ladder pattern of 160 - 180 bp or multiples thereof (oligonucleosomes) in cancer patients [8]. Apoptotic fragmentation of circulating DNA results from a caspase-activated DNase CUTTING free linker DNA between the nucleosome core particle ( $\sim 146 \mathrm{bp})$, which varies from $10-80 \mathrm{bp}$ in length depending on species and tissue type $[8,9]$. In addition, fragmentation occurred by lysosomal DNase II after the dying cells are phagocytosed [10]. Recent studies also demonstrated an additional cleavage of $10 \mathrm{bp}$ periodicity in relation to the helical pitch of nucleosome-bound cfDNA [11]. Contrary to the typical apoptotic size distribution, the work by Mouliere and colleagues $[12,13]$ described $K R A S$ mutated ctDNA fragments from colorectal cancer patients which were mainly smaller than $145-160 \mathrm{bp}$ as compared to circulating DNA from $K R A S$ wild type patients and nontumoral derived circulating DNA from healthy cells. In line, recent studies by Jiang [14], Snyder [11] and Underhill [15] et al. indicated a shift to smaller fragments in cancer patients. However, the dominant structure of circulating DNA was shown at $167 \mathrm{bp}$. With respect to preanalytic variables the choice of cfDNA extraction systems could influence detection of oligonucleosomes recently demonstrated by Pérez-Barrios and colleagues [16]. Furthermore, different extraction systems demonstrated variable cfDNA yields [17], showing the importance of pre-analytical studies to improve our knowledge of circulating DNA for routine clinical practice.

In this study, we assessed the impact of cfDNA and ctDNA yield on sensitive KRAS G12S and G12D detection in serum of CRC patients using promising Intplex allelespecific PCR (Intplex PCR). To go beyond previous pre-analytical efforts, we systematically compared an automated magnetic bead-based extraction system with a widely used membrane-based method showing a significant increased extraction of high molecular weight cfDNA fragments using the membrane-based kit in serum of colorectal cancer patients.

\section{RESULTS}

\section{Intplex PCR reveals an enhanced cfDNA quantity in serum of advanced CRC patients}

Today, the use of plasma instead of serum is recommended by many laboratories since the abundant cfDNA yield in serum, probably due to the enhanced lysis of circulating lymphocytes during serum preparation, reduces the relative amount of ctDNA. However, keeping in mind that clinically highly valuable patient collectives [18-22] are based on serum samples, we wondered if innovative Intplex PCR, first published by Thierry et al. [6], could be a reliable technology for sensitive analysis of oncological KRAS mutations in CRC patients' serum samples as well. To this effect, we decided to investigate the most frequently observed KRAS mutation in colorectal cancer patients, i.e. G12D with a frequency of 33.5 - 34.4\% among KRAS mutated colorectal cancers. In addition, we investigated G12S known to occur with low frequency of $4.9-5.7 \%$ in $K R A S$ mutated colorectal cancers.

First, we compared serum cfDNA amounts in colon cancer patients $(n=50)$ and healthy controls $(n=8)$ measured with the Qubit system and the independent Intplex PCR revealing highly comparable results (spearman r: 0.976, $\mathrm{P}<0.001$ ) (Figure 1A). Further, absolute cfDNA quantification of CRC serum samples with Intplex PCR indicated a significantly increased cfDNA yield in CRC patients compared to healthy controls (median cfDNA: $49.5 \mathrm{ng} / \mathrm{mL}$ serum) (Figure 1B). In more detail, in contrast to healthy controls median cfDNA concentration was 4.7-fold, 6.7-fold and 9.1-fold higher in patients with lymph node negative (median cfDNA: $231.7 \mathrm{ng} / \mathrm{mL}$ serum), lymph node positive (median cfDNA: $330.1 \mathrm{ng} / \mathrm{mL}$ serum) and those with distant metastasis disease (median cfDNA: $450.8 \mathrm{ng} / \mathrm{mL}$ serum), respectively.

Of interest, metastasized CRC patients showed increased cfDNA concentrations at occurrence of a second distant metastasis diagnosed six months $(n=2$, patient \#29 and \#38) and 13 months (n=1, patient \#37) after primary CRC (Figure 1C and 1D). In case of patient \#37 the first blood sample was drawn before surgery of the liver metastasis (serum 1) and the second 24 days later, before surgery of the primary colorectal tumor (serum 2). Interestingly, cfDNA amount decreased 2.7-fold after the first surgery and reached a nearly identical level at 
diagnosis of liver metastasis 13 months later (serum 3) (Figure 1D).

\section{Intplex PCR detects $K R A S$ mutations in serum of localized and advanced CRC patients}

Absolute quantification of KRAS G12D and G12S mutated ctDNA revealed a median concentration of 1.0 ng in $1 \mathrm{~mL}$ serum (Figure 2A). As expected, the highest amount of ctDNA was found in patients with distant organ metastasis. Allelic frequencies ranged from $0.02 \%$ to $2.65 \%$ with the highest median value $(0.46 \%)$ in metastasized patients diagnosed with a second metastasis (Figure 2B). However, ctDNA quantity or allele frequency did not enable discrimination of different CRC stages.

Overall, we identified 16 patients $(34.8 \%)$ who harbored either circulating mutant KRAS G12D ( $\mathrm{n}=11$; $23.9 \%)$ or G12S $(n=3 ; 6.5 \%)$ or both $(n=1 ; 2.2 \%)$. In more detail, $12.5 \%$ ( 3 of 24 serum samples were mutated) of patients with localized tumors revealed a KRAS mutation, while patients with lymph node positivity showed a KRAS mutation frequency of $44.0 \%$
(3 of 9 serum samples were $K R A S$ G12D and one sample was G12S mutated). A KRAS mutation frequency of 53\% (9 of 17 serum samples were mutated) was obtained in patients showing distant organ metastasis. Further, patient \#37 showed a G12D mutation at diagnosis of the first liver metastasis (see Figure 1D, serum 1) and at diagnosis of an additional liver metastasis 13 months after primary CRC diagnosis (see Figure 1D, serum 3). Interestingly, a $K R A S \mathrm{G12D}$ mutation was non-detectable in serum 2 (see Figure 1D) after surgery of the first liver metastasis. In addition, patient \#29 revealed a G12S mutation at diagnosis of liver metastasis (see Figure 1C).

We further investigated whether the obtained KRAS mutations in serum ctDNA were detectable in matched tissue DNA analyzed by Intplex PCR. Circulating KRAS mutations were not detected in 28 of 40 patients with $K R A S$ wild type primary tumors, yielding a specificity of $70 \%$. In addition, we identified five cases (of 10 ) in which mutations were present in the primary tumor tissue but not in serum ctDNA, yielding a sensitivity of $50 \%$. Concordance between KRAS mutation status in the serum and tumor tissue was $66 \%$. To strengthen Intplex PCR analysis, we investigated patients showing a KRAS
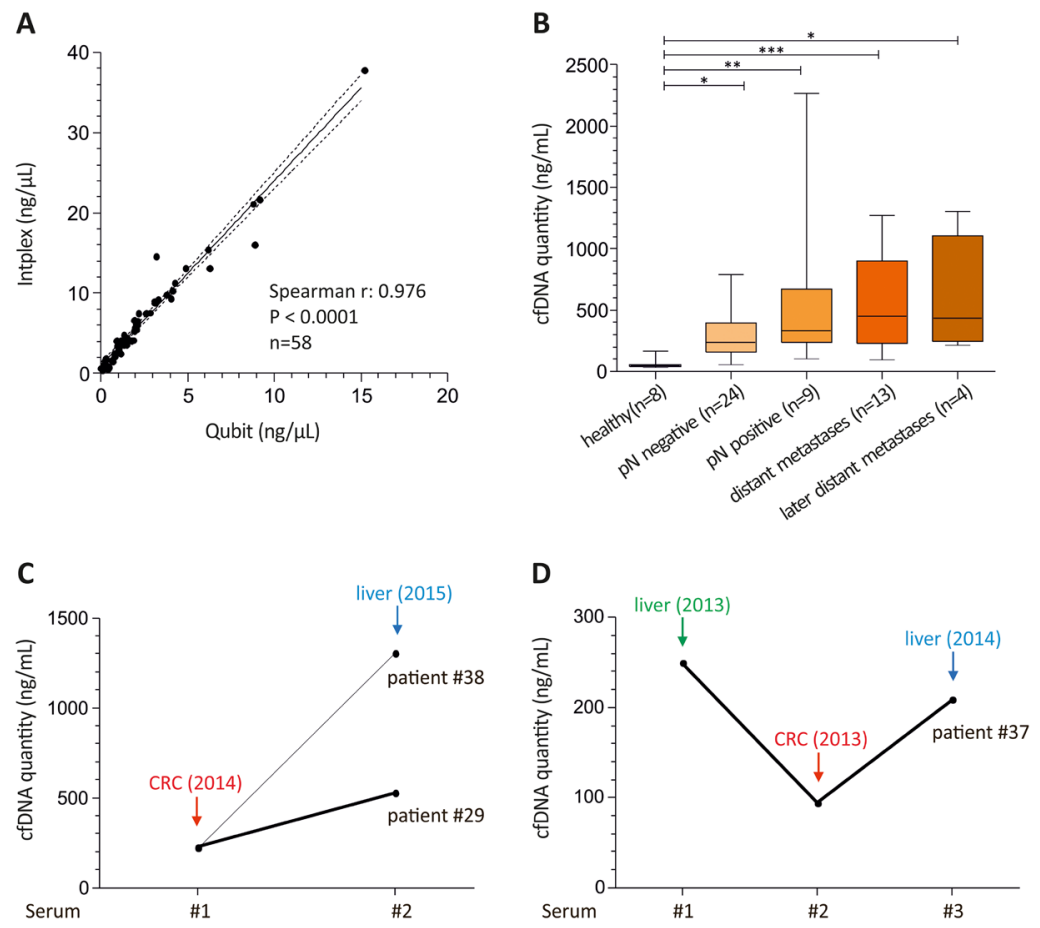

Figure 1: Intplex PCR shows abundant cfDNA levels in advanced colon cancer patients. (A) Spearman correlation of serum cfDNA amount in eight healthy and 50 serum samples from CRC patients revealed a strong association between Qubit and independent Intplex PCR measurement. (B) Box plot analysis indicated significant higher cfDNA concentrations in CRC patients compared to healthy individuals. (C) and (D) In patients with advanced cancer and additional distant metastasis six months (patient \#29 and \#38) and 13 months (patient \#37) after primary cancer diagnosis cfDNA concentrations were higher at the time point of second distant metastasis. In case of patient \#37 the first blood sample was drawn before surgery of the liver metastasis (serum 1) and the second 24 days later before surgery of the colorectal tumor (serum 2). Serum 3 was drawn at diagnosis of the liver metastasis 13 months later. Statistical analysis was performed using 1way ANOVA Kruskal-Wallis test to compare all groups where; ${ }^{*} \mathrm{P}<0.05,{ }^{*} \mathrm{P}<0.01,{ }^{*} * * \mathrm{P}<0.001$. 
G12D mutation in serum and/or tissue DNA by digital droplet PCR (ddPCR). Correlation analysis revealed a strong association (spearman $\mathrm{r}$ : $0.989, \mathrm{P}<0.001$ ) of wild type $K R A S$ allele copies measured by Intplex and ddPCR (Figure 2C). However, only 50\% (6 of 12 of KRAS G12D cases) was confirmed with ddPCR with similar copy numbers (Figure 2D and Supplementary Figure 1).

\section{The use of the silica-based membrane system promotes the extraction of high molecular weight cfDNA fragments}

Examined ctDNA concentrations and allelic frequencies were low. Therefore, we performed a more indepth investigation of pre-analytical technologies. Since most of liquid biopsy studies use silica membrane-based systems, in many cases in the presence of carrier RNA, we wondered whether carrier RNA may be of influence on cfDNA yield and sensitivity of $K R A S$ detection. A total of 38 serum samples from $10 \mathrm{CRC}$ patients (all stages) were subjected to cfDNA extraction using two independent systems, both demonstrating successful extraction of cfDNA (Supplementary Figure 2). To understand whether the use of carrier RNA may influence cfDNA yield and/ or absolute quantification of ctDNA with Intplex PCR we performed cfDNA extraction with and without the addition of $1 \mu \mathrm{g}$ carrier RNA of paired samples. Our data indicate no significant difference in absolute cfDNA and ctDNA yield depending on the addition of carrier RNA or extraction technology (Supplementary Figure 2A and B).

To go beyond cfDNA quantification with the Qubit system or Intplex PCR we additionally performed a cfDNA fragment analysis using Agilent's 2100 Bioanalyzer system. The silica-based membrane system showed a slightly increased quantity of low sized cfDNA fragments $(<600 \mathrm{bp}$ ) compared to the magnetic beads system. However, only the magnetic beads system revealed significant increased amounts of small cfDNA fragments compared to high molecular cfDNA fragments $(>600$ bp). Interestingly, the silica-based membrane system displayed a significant 2.5-fold higher concentration of high molecular cfDNA fragments ( $>600$ bp) (Figure $3 \mathrm{~A}$ and $3 \mathrm{~B}$ ). In line, the silica-based membrane system showed a significantly elevated percentage (normalized to total measured fragments) of $>600 \mathrm{bp}$ cfDNA fragments (Figure 3C and 3D), which are characteristic for cfDNA originating from lysed lymphocytes during blood processing. In contrast, the magnetic beads system showed a 1.6-fold increased percentage of extracted low sized
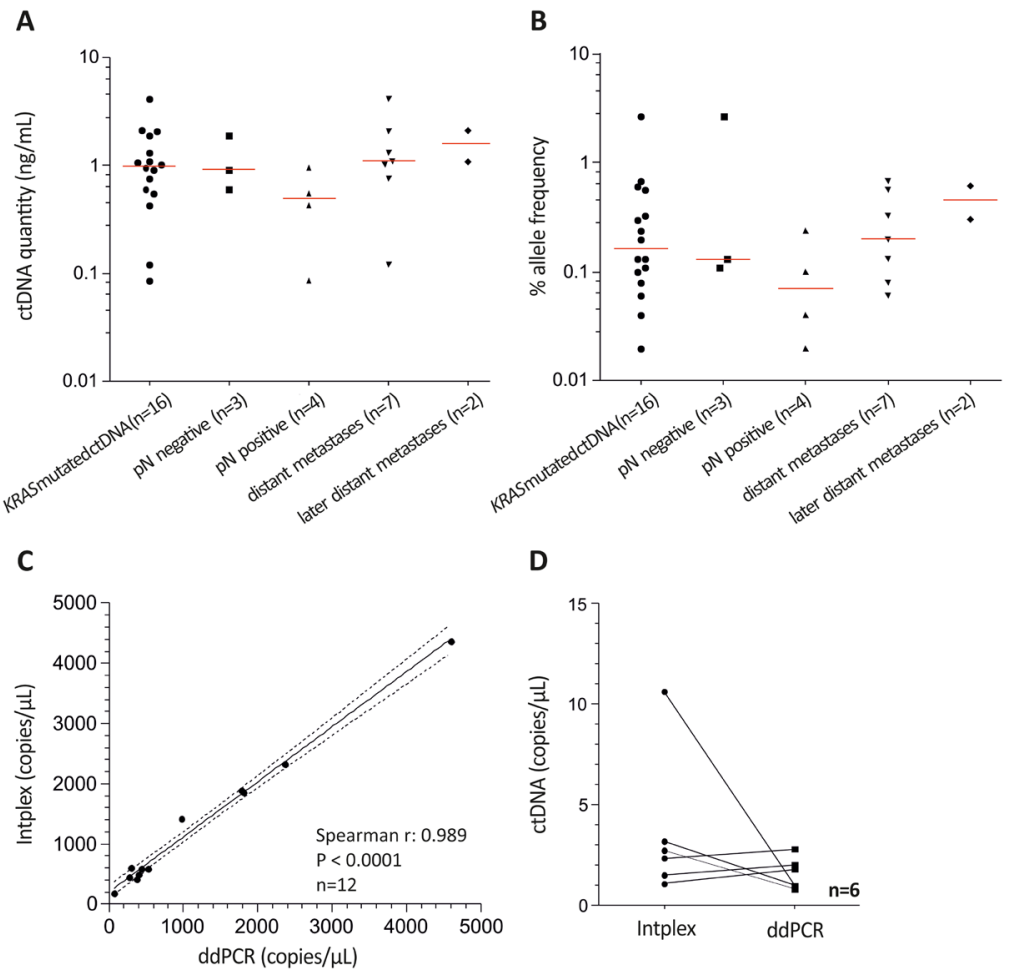

Figure 2: Intplex PCR indicates increased $K R A S$ allelic frequency in serum of metastasized CRC patients. (A) and (B) Scatter plot analysis indicated higher cfDNA concentrations and allelic frequencies in metastasized CRC compared to patients with local and lymph node positive healthy patients. Allelic frequencies ranging from $0.02 \%$ to $2.65 \%$ with the highest median value (0.46 $\%)$ in metastasized patients with a second diagnosed metastasis. (C) Spearman correlation of KRAS wild type allele copies revealed a strong association between ddPCR and independent Intplex PCR. (D) Measured KRAS G12D mutated allele copies were similar between Intplex and ddPCR. 
cfDNA fragments compared to silica-based membrane system (Figure 3C and 3D).

\section{The use of serum leads to a lower allelic frequency of $K R A S$ mutations compared to plasma}

Showing an increased yield of high molecular serum cfDNA fragments extracted using the silica-based membrane system we wondered if there is an influence on the detection of KRAS mutations depending on cfDNA-source. To shed light on this question, we next evaluated ctDNA and allelic frequency coverage using both extraction systems in plasma and serum samples from healthy individuals using spike-in DNA harboring a clinically relevant $K R A S$ G12D or G12S. As expected, absolute amounts of serum-cfDNA were higher compared to plasma-cfDNA levels: Based on two independent spike-in experiments a 6.7 -fold higher median cfDNA concentration was shown for the magnetic beads system, while the silica-based membrane system demonstrated 3.7-fold increased cfDNA yield in serum compared to plasma samples (Supplementary Figure 3).
Next, mean G12D and G12S spike-in DNA concentrations measured in serum were slightly higher as expected (Figure 4A). In contrast, absolute quantification of plasma spike-in DNA revealed a more accurate resemblance using the magnetic beads system, while with the silica-based membrane system significant higher concentrations were retrieved (Figure 4B). In addition, neither the samples without spike-in DNA nor KRAS wild type spike-in DNA gave an unspecific signal in serum or plasma. Based on absolute cfDNA and ctDNA quantification we calculated the allelic frequency of the mentioned KRAS mutations, which is expected to be $50 \%$ in all samples. Probably due to the elevated cfDNA levels, allelic frequencies were much lower than expected in serum samples compared to plasma (Figure 4C).

\section{DISCUSSION}

Today, molecular analysis of e.g. circulating tumor DNA in bodily fluids (also known as liquid biopsy) offers a supplementary option for accurate mutation testing in patients without available tumor tissue or to measure tumor burden early at disease relapse. Recent studies [2,
A

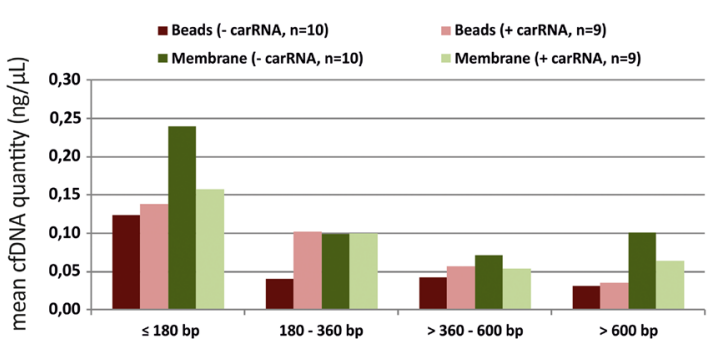

C

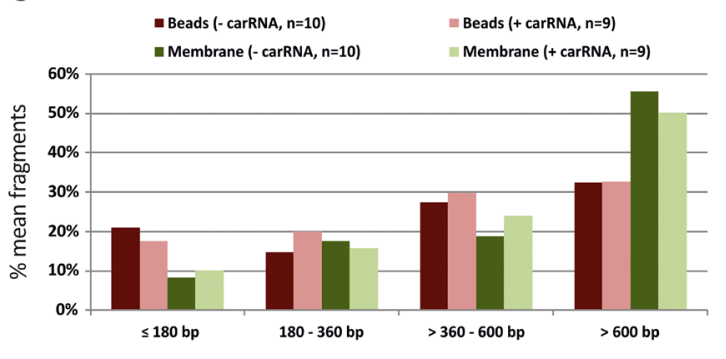

B

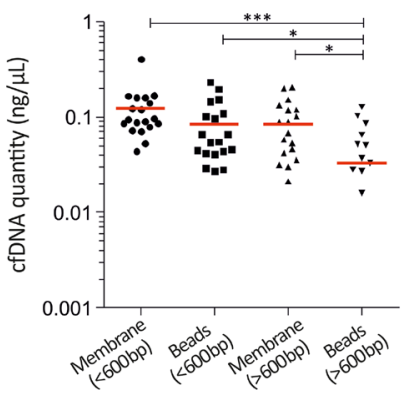

D

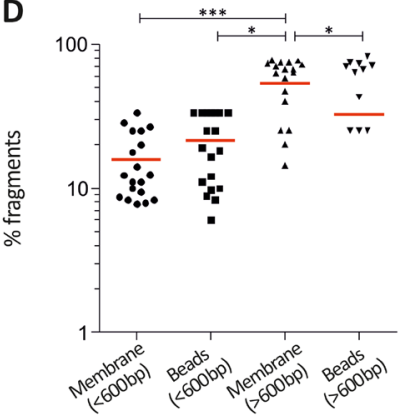

Figure 3: The use of the silica-based membrane system promotes the extraction of high molecular weight cfDNA fragments. (A) The silica-based membrane system revealed increased cfDNA yields mainly of small ( $\leq 180 \mathrm{bp})$ and high (>600 bp) fragments compared to the magnetic beads system. (B) Scatter plot analysis revealed a significant 2.5-fold increased concentration of high molecular cfDNA fragments ( $>600 \mathrm{bp}$ ) using the silica-based membrane system. In line, significant increased amounts of small cfDNA fragments $(<600 \mathrm{bp})$ compared to high molecular cfDNA fragments $(>600 \mathrm{bp})$ presented only with the magnetic beads system. (C) The silica-based membrane system showed reduced percentage (normalized to total measured fragments) of small cfDNA fragments compared to the magnetic beads system. (D) Scatter plot analysis indicated a significantly increased percentage of $>600$ bp cfDNA fragments using the silica-based membrane system. Histograms in (A) and (C) present mean values of $\mathrm{n}=18$ matched samples (extracted with carrier RNA) and $n=20$ matched samples (extracted without carrier RNA) from 10 CRC patients categorised in distinct fragment size groups. Red horizontal line in (B) and (D) shows mean of the mentioned groups. Statistical analysis was performed using 1way ANOVA Kruskal-Wallis test to compare all groups where; ${ }^{*} \mathrm{P}<0.05, * * * \mathrm{P}<0.001$. 
$3,6]$ showed the detection of clinically relevant oncologic driver mutations like e.g. KRAS, EGFR or secondary EGFR T790M resistance mutations in ctDNA with nearly maximal specificity (>95\%) compared to tumor tissue as the gold standard in cancer diagnostics. On the contrary, although digital PCR methods revealed a maximal technical detection limit of $0.01 \%$ (i.e. two mutated copies in a background of 20.000 wild type alleles), sensitivity of the mentioned mutations in ctDNA was unexpectedly low in some studies (ranging from 30 to $70 \%$ ) [7, 23]. Especially in early localized cancers the amount of detectable ctDNA is low [4]. In line, in the current study, of five KRAS G12D mutations found in tissue DNA of local lymph node negative CRC patients, only one mutation was detectable in matched serum ctDNA by Intplex PCR. Further, detection of mutated ctDNA in 10 patients with matched wild type tumor tissue DNA might be explained by tumor evolution during therapy, since seven of these patients were diagnosed with advanced stage CRC. In addition, it is obvious that more mutations are detected from ctDNA as compared to tumor tissue due to temporal, and intra-, or inter-clonal heterogeneity [4] resulting in a moderate concordance. Of importance, ctDNA mutations in serum with paired wild type tissue DNA could be an early indicator for tumor relapse or non- detectable (micro-) metastasis, since recent studies showed the application of ctDNA in monitoring of colorectal [24] and breast [25] cancer patients.

Unfortunately, therapy- or clinical follow-up data of the analyzed patients were not available. However, only $50 \%$ of Intplex KRAS G12D cases confirmed with only ddPCR. In addition, two cases with an Intplex KRAS ctDNA wild type indicated as mutated with ddPCR. Keeping in mind that KRAS mutated ctDNA from colorectal cancer patients showed with a high quantity of low-size fragments $(<80 \mathrm{bp})$ [13] discrepancy of mutational status might be explained by different primer amplicon length. While Intplex PCR amplified a $60 \mathrm{bp}$ sequence of the $K R A S$ allele, ddPCR uses a 90 bp primer amplicon. However, a strong association (spearman r: $0.976, \mathrm{P}<0.001$ ) of wild type $K R A S$ allele copies measured with Intplex and ddPCR was found, underlining robustness and reliability of Intplex PCR.

Besides the biological ctDNA complexity the source of ctDNA, i.e. the use of serum in our study, could be a reason for lower sensitivity or discrepancy between Intplex and ddPCR. While many retrospective and clinically highly significant patient cohorts still rely on serum samples we addressed the reliability of the Intplex PCR technology in a comprehensive CRC serum
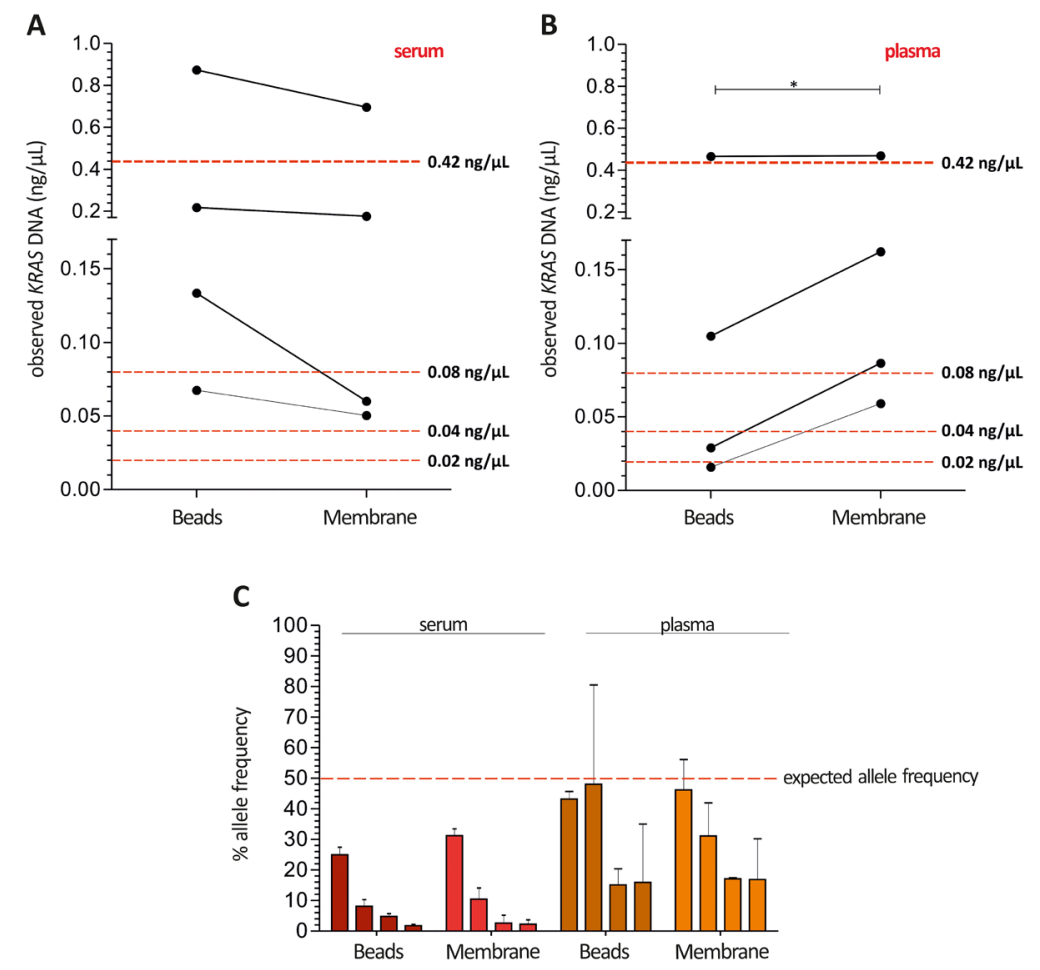

Figure 4: Higher cfDNA yields in serum may result in a lower allelic frequency of $K R A S$ mutations compared to plasma. Median KRAS G12D and G12S ctDNA concentrations measured in serum (A) were slightly higher as the expected concentrations, while absolute ctDNA quantification in plasma (B) represents the amounts of spike-in DNA with better accuracy showing the best suitable quantities for the magnetic beads system (red dotted line: expected ctDNA yield). (C) Allelic frequencies were much lower than expected in serum samples compared to plasma using Intplex PCR (red dotted line: expected allele frequency). Expected ctDNA yield calculated concerning the spike-in DNA concentrations $50 \mathrm{ng} / \mu \mathrm{L}-10 \mathrm{ng} / \mu \mathrm{L}-5 \mathrm{ng} / \mu \mathrm{L}-2.5 \mathrm{ng} / \mu \mathrm{L}$ eluted in $60 \mu \mathrm{L}$ elution buffer and $50 \%$ allele frequency. Statistical analysis was performed using a paired Student's t-test where; $* \mathrm{P}<0.05$. 
Table 1: Histo-pathological patient characteristics

\begin{tabular}{|c|c|c|}
\hline Categorisation & Patients $\left(\mathrm{n}^{\mathrm{a}}=\mathbf{5 0}\right)$ & $\%$ \\
\hline Median age at diagnosis (range) & 73 years $(43-89)$ & \\
\hline \multicolumn{3}{|l|}{ Gender } \\
\hline female & 23 & $46 \%$ \\
\hline male & 27 & $54 \%$ \\
\hline \multicolumn{3}{|l|}{ Localisation } \\
\hline right-sided & 15 & $30 \%$ \\
\hline left-sided & 31 & $62 \%$ \\
\hline unknown & 4 & $8 \%$ \\
\hline \multicolumn{3}{|l|}{ Histological grade } \\
\hline G2 & 40 & $80 \%$ \\
\hline G3 & 6 & $12 \%$ \\
\hline unknown & 4 & $8 \%$ \\
\hline \multicolumn{3}{|l|}{ pT } \\
\hline pT 1-2 & 13 & $26 \%$ \\
\hline pT 3-4 & 34 & $68 \%$ \\
\hline unknown & 3 & $6 \%$ \\
\hline \multicolumn{3}{|l|}{$\mathbf{p N}$} \\
\hline $\mathrm{pN}$ negative & 28 & $56 \%$ \\
\hline $\mathrm{pN}$ positive & 19 & $38 \%$ \\
\hline unknown & 3 & $6 \%$ \\
\hline \multicolumn{3}{|l|}{$\mathbf{p M}$} \\
\hline pM positive & 14 & $28 \%$ \\
\hline pM unknown & 36 & $52 \%$ \\
\hline \multicolumn{3}{|l|}{ Localisation distant metastases } \\
\hline liver & 16 & \\
\hline kidney & 2 & \\
\hline ovary & 1 & \\
\hline peritoneum & 1 & \\
\hline omentum majus & 1 & \\
\hline colon & 4 & \\
\hline
\end{tabular}

collective. Comparing circulating mutational load of $K R A S$ G12D and G12S in distant metastasized CRC patients in our study to the work by Thierry et al. [6] revealed a 5.2fold and 48.7-fold decrease in median ctDNA yield and allelic frequency, respectively. As expected, total cfDNA was 7.6-fold higher in our serum-based study compared to plasma analysed by Thierry et al. probably resulting in a lower ctDNA yield and allele frequency of detected $K R A S$ mutations in our study. In line, we demonstrated a higher median cfDNA concentration using the magnetic beads or silica-based membrane system in serum compared to plasma samples of healthy individuals. Further spike-in experiments clearly demonstrated that a higher cfDNA yield in serum decreased the measurement of ctDNA allele frequencies with Intplex PCR independent of the used extraction technology. 
Today, some research studies have been seeking to improve the pre-analytical steps of ctDNA processing by using specialized cell free DNA blood stabilization tubes $[17,26,27]$. In addition, different extraction methods for the isolation of cfDNA from serum or plasma samples were compared demonstrating that the extraction method can considerably affect cfDNA quantity $[16,17,28]$. Similarly, the current study observed that both silica- and magnetic beads-based methods yielded sufficient cfDNA for downstream PCR applications, while the magnetic beads system (used in this study) is a simpler and more rapid automated method. Further, similar absolute cfDNA and ctDNA yields with both technologies and conditions (+ and - carrier RNA) were observed. Interestingly, the use of a silica-based membrane system enhances the extraction of long cfDNA rather than shorter ctDNA fragments, in serum of CRC patients. Notably, the magnetic beads system more efficiently recovers both short and fragmented cfDNA, probably representing circulating DNA from apoptotic tumor cells. This observation should be validated in more patients since it has been suggested that low molecular weight cfDNA fractions often harbor genetic aberrations indicative of tumor-derived DNA [13, 15]. Concerning fragment size analysis, one should be aware that the 2100 Bioanalyzer from Agilent revealed a more accurate fragment size recovery compared to the TapeStation (Agilent) system (Supplementary Figure 4).

To this end, careful consideration remains necessary for optimal cfDNA extraction, fragment size analysis and specific ctDNA amplification in liquid biopsy. Intplex allele-specific PCR is a sensitive technology for the absolute measurement of total cfDNA as well as detection of oncological driver mutations even in serum samples while reducing cost and time of analysis. Further, we demonstrated that there are significant differences in high and low molecular weight cfDNA fractions recovery according to the methodologies evaluated. A limitation of this study is the small number of samples studied $(n=38$ paired samples from $10 \mathrm{CRC}$ patients) while larger studies are needed to evaluate the impact of such differences on downstream PCR applications in routine clinical practice. Underlining recent investigations, plasma should be the analyte of choice in liquid biopsy analyses. However, using the best suited (pre-) analytical system clinically valuable retrospective serum cohorts can be investigated with clinical purpose as well.

\section{MATERIALS AND METHODS}

\section{Patients}

Subject of this study was a cohort of 50 colorectal cancer patients including FFPE tissues and serum samples. Hematoxylin and eosin-stained sections of FFPE tissues were prepared for assessment of the percentage of tumor cells by a pathologist. The analyzed patient cohort represents various stages of colon cancer tumorigenesis compromising lymph node negative and distant metastasis negative cases $(\mathrm{n}=24)$, lymph node positive and distant metastasis negative cases $(n=10)$, lymph node negative or positive and distant metastasis positive cases $(\mathrm{n}=16)$ and the metastases tissues $(n=26)$ from different organ sites at different time points (i.e. metastases tissue at primary CRC diagnosis and at later distant metastasis).

For ctDNA analysis matched serum samples were available from 46 patients at primary cancer diagnosis including four patients with an additional serum sample at diagnosis of subsequent metastasis. Biomaterial samples were provided by the RWTH centralized Biomaterial Bank Aachen (RWTH cBMB, Aachen, Germany) in accordance with the regulations of the biomaterial bank and the approval of the ethics committee of the medical faculty, RWTH Aachen. Eight serum samples from healthy individuals were included as controls. All patients gave written informed consent for retention and analysis of their tissue for research purposes (local ethical review board of the medical faculty of the RWTH Aachen, ref no. EK-206/09). An overview of patients' characteristics is given in Table 1. Tissue and paired serum samples were collected between 2012 and 2015. Blood was drawn prior to tumor tissue removal.

\section{Sample processing}

Blood samples $(10 \mathrm{~mL})$ from all study participants were obtained by venipuncture. All samples were processed at room temperature within $3 \mathrm{~h}$ from the time of blood extraction. Hemolyzed samples were discarded for further analysis. For serum preparation blood was collected in tubes containing a clot activator (S-Monovette, Order no. 02.1063, Sarstedt, Nümbrecht, Germany). According to manufacturer's protocol samples were centrifuged at $2.000 \times \mathrm{g}$ for 10 minutes at room temperature. Plasma was prepared from whole blood collected in K3 EDTA tubes (S-Monovette, Order no. 02.1066.001, Sarstedt, Nümbrecht, Germany) at $2.000 \mathrm{x} g$ for 10 minutes at room temperature according to manufacturer's recommendation. The resulting supernatant (either serum or plasma) was carefully aspirated from the tube (in case of plasma without disturbing the buffy coat layer) and transferred in $1 \mathrm{~mL}$ aliquots into $1.5 \mathrm{~mL}$ tubes, and then centrifuged a second time at $16.000 \mathrm{x}$ g for 10 minutes to remove cellular debris. Serum or plasma aliquots were then transferred to a new $1.5 \mathrm{~mL}$ tube and stored at $-80^{\circ} \mathrm{C}$ until use. At the time when the cfDNA extraction was performed samples were thawed once on ice. No freezethaw cycles of analysed blood samples was done.

\section{DNA extraction from FFPE tissue}

DNA was extracted from $5 \times 10 \mu \mathrm{m}$ freshly-cut FFPE tissue sections using the QIAamp DNA Mini kit 
(Qiagen, Hilden, Germany) according to manufacturer's protocol. DNA was eluted in $200 \mu \mathrm{l}$ of elution buffer.

\section{DNA extraction from plasma and serum}

A comparison of two extraction kits was performed with 38 paired serum samples from 10 colon cancer patients (metastatic or not) as well as plasma or serum from healthy donors with distinct concentrations of spikein DNA. The following kits were used: Maxwell RSC ccfDNA Plasma kit (Promega, Madison, USA; designated in the manuscript as magnetic beads system) and QIAamp Free Circulating Nucleic Acid kit (Qiagen, Hilden, Germany; designated in the manuscript as silica-based membrane system). Samples were processed according to the manufacturers' protocols with slight modifications: Serum samples from paired CRC patients were isolated using the magnetic beads system or the silica-based membrane system either with $(+1 \mu \mathrm{g}$ carrier RNA) or without the addition of carrier RNA (- carrier RNA).

For spike-in experiments DNA exhibiting a KRAS G12S or G12D mutation (Horizon Discovery, Cambridge, United Kingdom; Batch: 16118 (G12D) and 20815 (G12S)) with an allelic frequency of $50 \%$ was used. A $K R A S$ wild type DNA (Horizon Discovery, Cambridge, United Kingdom; allelic frequency 100\%; Batch: 17605) was used as control. Serum or plasma without spike-in DNA was used as an additional negative control. Besides the original DNA concentration $(50 \mathrm{ng} / \mu \mathrm{L})$, spikein DNA was diluted with $10 \mathrm{mM}$ Tris Buffer to a final concentration of $25 \mathrm{ng}, 5 \mathrm{ng}$ and $2.5 \mathrm{ng}$ of which $1 \mu \mathrm{L}$ was spiked into $1 \mathrm{~mL}$ serum or plasma from healthy donors. Spike-in DNA was extracted with the magnetic bead or the silica membrane system according to the manufacturers' protocols (magnetic beads system without carrier RNA, silica-based membrane system with carrier RNA) following absolute DNA quantification by Intplex PCR.

In all cases, cfDNA was isolated using as starting volume $1 \mathrm{~mL}$ of serum or plasma, eluted in $60 \mu \mathrm{L}$ of the supplied elution buffer and stored at $-80^{\circ} \mathrm{C}$ until use.

\section{Agilent 2100 bioanalyzer}

Fragment analysis was performed at the Genomicsfacility of the Interdisciplinary Center for Clinical Research (IZKF) at the university hospital Aachen. The High Sensitivity DNA Assay on the 2100 Bioanalyzer enabled analysis of cfDNA fragments between 35 and $10.000 \mathrm{bp}$, according to manufacturer's protocol.

\section{Intplex allele-specific PCR}

Intplex allele-specific PCR was performed according to the protocol by Thierry et al. [6] with modifications: qPCR amplifications were carried out in triplicate in a reaction volume of $25 \mu \mathrm{L}$ on an IQ5 instrument using iQ5 Optical system software 2.0 (Bio-Rad, Munich,
Germany). The $25 \mu \mathrm{L}$ qPCR mix was composed of 12.5 $\mu \mathrm{L}$ of GoTaq qPCR Master Mix (Promega), $0.75 \mu \mathrm{L}$ of each amplification primer $(0.3 \mathrm{pmol} / \mu \mathrm{L}), 9.25 \mu \mathrm{l}$ of nucleic acid-free water (Promega) and $1 \mu \mathrm{L}$ of extracted cfDNA. Primer sequences, thermal cycling, melting curves and data analysis were investigated as mentioned by Thierry et al. [6]. To ensure specific detection of the targeted mutation, each run contained positive and negative controls. Positive control DNA was extracted from cell lines SK-LU-1 (confirmed G12D mutation) and A546 (confirmed G12S mutation). Serial dilutions of genomic cell line DNA were used as a standard for absolute quantification. $K R A S$ wild type DNA was used as a negative control and autoclaved water was used as a non-template control.

\section{Digital droplet PCR}

The Bio-Rad QX200 System was used for digital droplet PCR (ddPCR). The ddPCR reaction mixture was loaded into the emulsification device and droplets were formed following the manufacturer's instructions. The contents were transferred to a 96-well reaction plate and sealed with a pre-heated Eppendorf 96-well heat sealer for 2 seconds. Total DNA was amplified separately in a C1000 Touch Thermal Cycler (Bio-Rad). The ddPCRTM probe assay KRAS p.G12D, Human (Unique Assay ID: $\mathrm{dHsaCP} 2500596)$ was used for analysis. Each reaction consisted of a $20 \mu \mathrm{L}$ solution containing $10 \mu \mathrm{L}$ ddPCR Probe Supermix, $450 \mathrm{nM}$ primers, $250 \mathrm{nM}$ probe, and 1 $\mu \mathrm{L}$ template DNA with the following cycling conditions: 10 minutes at $95^{\circ} \mathrm{C}, 40$ cycles each consisting of a 30 second denaturation at $94^{\circ} \mathrm{C}$ followed by a $55^{\circ} \mathrm{C}$ extension for 60 seconds, and a final 10 minutes at $98^{\circ} \mathrm{C}$. After cycling droplets were analyzed immediately. Absolute quantities of mutant and wild type $K R A S$ cfDNA copies were determined using the QuantaSoft software. Briefly, the system uses a 2-color detection system for the wild type (HEX) and mutant (FAM) alleles to count the number of droplets positive for each fluorophore. We considered samples as positive for mutant KRAS when 1 positive FAM droplet were identified above the threshold level.

\section{Statistical analysis}

Statistical analyses were performed using SPSS 22.0 (SPSS, Chicago, IL) and GraphPad Prism 5.0 (GraphPad Software Inc., La Jolla, CA). Box Plot graphs are shown as follows: Horizontal lines: grouped medians. Boxes: $25-75 \%$ quartiles. Vertical lines: range, peak and minimum. The 1way ANOVA Kruskal-Wallis test was used to compare cfDNA concentrations between distinct conditions. Correlation analysis was performed by calculating a Spearman correlation coefficient. Differences were considered statistically significant if the two-sided p-values were equal or below $5 \%(\leq 0.05)$. 


\section{Abbreviations}

bp: base pairs; cfDNA: free circulating DNA; CRC: colorectal Cancer, ctDNA: circulating tumor DNA; ddPCR: digital droplet PCR; EGFR: Epidermal Growth Factor Receptor; KRAS: Kirsten rat sarcoma viral oncogene homolog; NSCLC: non-small cell lung cancer; PCR: polymerase chain reaction.

\section{Author contributions}

$\mathrm{VK}, \mathrm{RK}$ and ED participated in the design of the study. VK, NR, NOB, JG, NF, JE and JM performed the experimental data acquisition. VK and NR performed data analyses. FS assessed tumor tissues. MB provided serum samples. VK wrote the manuscript. All authors read, critically revised, and approved the final manuscript.

\section{ACKNOWLEDGMENTS}

The excellent support by the RWTH centralized Biomaterial Bank Aachen (RWTH cBMB) of the University Hospital RWTH Aachen is thankfully acknowledged.

\section{CONFLICTS OF INTEREST}

There are no potential conflicts of interest.

\section{REFERENCES}

1. Forshew T, Murtaza M, Parkinson C, Gale D, Tsui DW, Kaper F, Dawson SJ, Piskorz AM, Jimenez-Linan M, Bentley D, Hadfield J, May AP, Caldas C, et al. Noninvasive identification and monitoring of cancer mutations by targeted deep sequencing of plasma DNA. Sci Trans1 Med. 2012; 4: 136ra68

2. Murtaza M, Dawson SJ, Tsui DW, Gale D, Forshew T, Piskorz AM, Parkinson C, Chin SF, Kingsbury Z, Wong AS, Marass F, Humphray S, Hadfield J, et al. Non-invasive analysis of acquired resistance to cancer therapy by sequencing of plasma DNA. Nature. 2013; 497: 108-12.

3. Oxnard GR, Paweletz CP, Kuang Y, Mach SL, O'Connell A, Messineo MM, Luke JJ, Butaney M, Kirschmeier P, Jackman DM, Janne PA. Noninvasive detection of response and resistance in EGFR-mutant lung cancer using quantitative next-generation genotyping of cell-free plasma DNA. Clin Cancer Res. 2014; 20: 1698-705.

4. Bettegowda C, Sausen M, Leary RJ, Kinde I, Wang Y, Agrawal N, Bartlett BR, Wang H, Luber B, Alani RM, Antonarakis ES, Azad NS, Bardelli A, et al. Detection of circulating tumor DNA in early- and late-stage human malignancies. Sci Trans1 Med. 2014; 6: 224ra24.

5. Hindson BJ, Ness KD, Masquelier DA, Belgrader P, Heredia NJ, Makarewicz AJ, Bright IJ, Lucero MY,
Hiddessen AL, Legler TC, Kitano TK, Hodel MR, Petersen JF, et al. High-throughput droplet digital PCR system for absolute quantitation of DNA copy number. Anal Chem. 2011; 83: 8604-10.

6. Thierry AR, Mouliere F, El MS, Mollevi C, Lopez-Crapez E, Rolet F, Gillet B, Gongora C, Dechelotte P, Robert B, Del RM, Lamy PJ, Bibeau F, et al. Clinical validation of the detection of KRAS and BRAF mutations from circulating tumor DNA. Nat Med. 2014; 20: 430-5.

7. Karlovich C, Goldman JW, Sun JM, Mann E, Sequist LV, Konopa K, Wen W, Angenendt P, Horn L, Spigel D, Soria JC, Solomon B, Camidge DR, et al. Assessment of EGFR mutation status in matched plasma and tumor tissue of NSCLC patients from a phase I study of rociletinib (CO1686). Clin Cancer Res. 2016; 22: 2386-95.

8. Jahr S, Hentze H, Englisch S, Hardt D, Fackelmayer FO, Hesch RD, Knippers R. DNA fragments in the blood plasma of cancer patients: quantitations and evidence for their origin from apoptotic and necrotic cells. Cancer Res. 2001; 61: 1659-65.

9. Thierry AR, El Messaoudi S, Gahan PB, Anker P, Stroun M. Origins, structures, and functions of circulating DNA in oncology. Cancer Metastasis Rev. 2016; 35: 347-76.

10. Nagata S, Nagase H, Kawane K, Mukae N, Fukuyama H. Degradation of chromosomal DNA during apoptosis. Cell Death Differ. 2003; 10: 108-16.

11. Snyder MW, Kircher M, Hill AJ, Daza RM, Shendure J. Cell-free DNA comprises an in vivo nucleosome footprint that informs its tissues-of-origin. Cell. 2016; 164: 57-68.

12. Mouliere F, El Messaoudi S, Gongora C, Guedj AS, Robert B, Del Rio M, Molina F, Lamy PJ, Lopez-Crapez E, Mathonnet M, Ychou M, Pezet D, Thierry AR. Circulating cell-free DNA from colorectal cancer patients may reveal high KRAS or BRAF mutation load. Transl Oncol. 2013; 6: 319-28.

13. Mouliere F, Robert B, Arnau Peyrotte E, Del Rio M, Ychou M, Molina F, Gongora C, Thierry AR. High fragmentation characterizes tumour-derived circulating DNA. PLoS One. 2011; 6: e23418.

14. Jiang $\mathrm{P}$, Chan $\mathrm{CW}$, Chan $\mathrm{KC}$, Cheng SH, Wong J, Wong VW, Wong GL, Chan SL, Mok TS, Chan HL, Lai PB, Chiu RW, Lo YM. Lengthening and shortening of plasma DNA in hepatocellular carcinoma patients. Proc Natl Acad Sci U S A. 2015; 112: E1317-E25.

15. Underhill HR, Kitzman JO, Hellwig S, Welker NC, Daza R, Baker DN, Gligorich KM, Rostomily RC, Bronner MP, Shendure J. Fragment length of circulating tumor DNA. PLoS Genet. 2016; 12: e1006162.

16. Perez-Barrios C, Nieto-Alcolado I, Torrente M, JimenezSanchez C, Calvo V, Gutierrez-Sanz L, Palka M, DonosoNavarro E, Provencio M, Romero A. Comparison of methods for circulating cell-free DNA isolation using blood from cancer patients: impact on biomarker testing. Transl Lung Cancer Res. 2016; 5: 665-72. 
17. Sherwood JL, Corcoran C, Brown H, Sharpe AD, Musilova M, Kohlmann A. Optimised pre-analytical methods improve KRAS mutation detection in circulating tumour DNA (ctDNA) from patients with non-small cell lung cancer (NSCLC). PLoS One. 2016; 11: e0150197.

18. Diaz LA Jr, Williams RT, Wu J, Kinde I, Hecht JR, Berlin J, Allen B, Bozic I, Reiter JG, Nowak MA, Kinzler KW, Oliner KS, Vogelstein B. The molecular evolution of acquired resistance to targeted EGFR blockade in colorectal cancers. Nature. 2012; 486: 537-40.

19. Jansen MP, Martens JW, Helmijr JC, Beaufort CM, van MR, Krol NM, Monkhorst K, Trapman-Jansen AM, Meijer-van Gelder ME, Weerts MJ, Ramirez-Ardila DE, Dubbink HJ, Foekens JA, et al. Cell-free DNA mutations as biomarkers in breast cancer patients receiving tamoxifen. Oncotarget. 2016; 7: 43412-8. https://doi.org/10.18632/oncotarget.9727.

20. Kinugasa H, Nouso K, Tanaka T, Miyahara K, Morimoto Y, Dohi C, Matsubara T, Okada H, Yamamoto K. Droplet digital PCR measurement of HER2 in patients with gastric cancer. Br J Cancer. 2015; 112: 1652-5.

21. Kloten V, Becker B, Winner K, Schrauder MG, Fasching PA, Anzeneder T, Veeck J, Hartmann A, Knuchel R, Dahl E. Promoter hypermethylation of the tumor-suppressor genes ITIH5, DKK3, and RASSF1A as novel biomarkers for blood-based breast cancer screening. Breast Cancer Res. 2013; 15: R4.

22. Oshiro C, Kagara N, Naoi Y, Shimoda M, Shimomura A, Maruyama N, Shimazu K, Kim SJ, Noguchi S. PIK3CA mutations in serum DNA are predictive of recurrence in primary breast cancer patients. Breast Cancer Res Treat. 2015; 150: 299-307.
23. Thress KS, Brant R, Carr TH, Dearden S, Jenkins S, Brown $\mathrm{H}$, Hammett T, Cantarini M, Barrett JC. EGFR mutation detection in ctDNA from NSCLC patient plasma: a crossplatform comparison of leading technologies to support the clinical development of AZD9291. Lung Cancer. 2015; 90: 509-15.

24. Reinert T, Scholer LV, Thomsen R, Tobiasen H, Vang S, Nordentoft I, Lamy P, Kannerup AS, Mortensen FV, Stribolt K, Hamilton-Dutoit S, Nielsen HJ, Laurberg S, et al. Analysis of circulating tumour DNA to monitor disease burden following colorectal cancer surgery. Gut. 2016; 65: 625-34.

25. Garcia-Murillas I, Schiavon G, Weigelt B, Ng C, Hrebien S, Cutts RJ, Cheang M, Osin P, Nerurkar A, Kozarewa I, Garrido JA, Dowsett M, Reis-Filho JS, et al. Mutation tracking in circulating tumor DNA predicts relapse in early breast cancer. Sci Transl Med. 2015; 7: 302ra133.

26. Kang Q, Henry NL, Paoletti C, Jiang H, Vats P, Chinnaiyan AM, Hayes DF, Merajver SD, Rae JM, Tewari M. Comparative analysis of circulating tumor DNA stability In K3EDTA, Streck, and CellSave blood collection tubes. Clin Biochem. 2016; 49: 1354-60.

27. Parpart-Li S, Bartlett B, Popoli M, Adleff V, Tucker L, Steinberg R, Georgiadis A, Phallen J, Brahmer JR, Azad NA, Browner I, Laheru DA, Velculescu VE, et al. The effect of preservative and temperature on the analysis of circulating tumor DNA. Clin Cancer Res. 2017; 23: 2471-7.

28. Fong SL, Zhang JT, Lim CK, Eu KW, Liu Y. Comparison of 7 methods for extracting cell-free DNA from serum samples of colorectal cancer patients. Clin Chem. 2009; 55: 587-9. 